\title{
The impact of hybrid neurosurgeons on the outcomes of endovascular coiling for unruptured cerebral aneurysms
}

\author{
Kimon Bekelis, MD, ${ }^{1}$ Dan Gottlieb, MS, ${ }^{2}$ Nicos Labropoulos, PhD, ${ }^{3}$ Yin Su, MS, ${ }^{2}$ \\ Stavropoula Tjoumakaris, MD, ${ }^{4}$ Pascal Jabbour, MD, ${ }^{4}$ and Todd A. MacKenzie, PhD $2,5,6,7$
}

${ }^{1}$ Section of Neurosurgery, Dartmouth-Hitchcock Medical Center, Lebanon; ${ }^{2}$ The Dartmouth Institute for Health Policy and Clinical Practice, Lebanon, New Hampshire; ' ${ }^{3}$ Department of Radiology, Stony Brook University Medical Center, Stony Brook, New York; ${ }^{4}$ Department of Neurosurgery, Jefferson Hospital for Neuroscience, Philadelphia, Pennsylvania; ${ }^{5}$ Department of Biomedical Data Science, Geisel School of Medicine at Dartmouth, Hanover; ${ }^{6}$ Department of Medicine, Dartmouth-Hitchcock Medical Center, Lebanon; and 'Department of Community and Family Medicine, Dartmouth-Hitchcock Medical Center, Lebanon, New Hampshire

OBJECTIVE The impact of combined practices on the outcomes of unruptured cerebral aneurysm coiling remains an issue of debate. The authors investigated the association of combined open and endovascular expertise with the outcomes of unruptured cerebral aneurysm coiling.

METHODS The authors performed a cohort study of $100 \%$ of Medicare fee-for-service claims data for elderly patients who underwent endovascular coiling for unruptured cerebral aneurysms between 2007 and 2012. To control for confounding, the authors used propensity score conditioning, with mixed effects to account for clustering at the hospital referral region level.

RESULTS During the study period, there were 11,716 patients who underwent endovascular coiling for unruptured cerebral aneurysms and met the inclusion criteria. Of these, $1186(10.1 \%)$ underwent treatment performed by hybrid neurosurgeons, and 10,530 (89.9\%) by proceduralists who performed only endovascular coiling. Multivariable regression analysis with propensity score adjustment demonstrated a lack of association of combined practice with 1-year postoperative mortality (OR 0.84; 95\% Cl 0.58-1.23), discharge to rehabilitation (OR 1.0; 95\% Cl 0.66-1.51), 30-day readmission

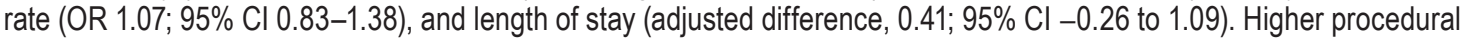
volume was independently associated with improved outcomes.

CONCLUSIONS In a cohort of Medicare patients, the authors did not demonstrate a difference in mortality, discharge to rehabilitation, readmission rate, and LOS between hybrid neurosurgeons and proceduralists performing only endovascular coiling.

http://thejns.org/doi/abs/10.3171/2015.11.JNS151725

KEY WORDS cerebral aneurysms; endovascular surgery; elderly; hybrid neurosurgeons; Medicare; vascular disorders; interventional neurosurgery

$\mathrm{C}$ EREBRAL aneurysm rupture is a devastating event. ${ }^{1,4}$ There are 2 distinct treatment options for unruptured aneurysms to prevent this outcome..$^{111}$ Clipping involves a craniotomy and placement of a clip on the blood vessel to exclude the weakened area, whereas endovascular coiling is a minimally invasive endovascular surgical approach achieving aneurysm obliteration from within the blood vessel. ${ }^{1,4}$ Although neurosurgeons in the United States performed clipping almost exclusively, the training paradigm has changed dramatically in recent years, with a growing number of hybrid neurosurgeons performing both clipping and coiling. ${ }^{1}$ Endovascular coiling is also performed by radiologists and other proceduralists who solely focus on this approach for the treatment of cerebral aneurysms. It is often questioned whether hybrid neurosurgeons can perform endovascular surgery as successfully and safely as providers focusing only on coiling.

However, limited literature exists that has attempted to answer this question. De Vries and Boogaarts published a single-center series ${ }^{6}$ on the outcomes of coiling of ruptured cerebral aneurysms by 2 hybrid neurosurgeons. In their single-center study, they did not use a comparison

ABBREVIATIONS COPD = chronic obstructive pulmonary disease; $L O S$ = length of stay.

SUBMITTED July 23, 2015. ACCEPTED November 23, 2015.

INCLUDE WHEN CITING Published online February 26, 2016; DOI: 10.3171/2015.11.JNS151725. 
group and did not employ multivariable techniques to control for confounding. Therefore their results cannot be generalized, or compared with those of proceduralists with expertise only with endovascular coiling. No prior study has investigated the association of combined open and endovascular expertise on the outcomes of unruptured cerebral aneurysm coiling in a national comprehensive cohort of elderly patients.

We performed a cohort study of Medicare patients with unruptured cerebral aneurysms to investigate the association of combined open and endovascular expertise on the outcomes of endovascular coiling. The outcomes examined were 1-year mortality, 30-day readmission, length of stay (LOS), and discharge to a rehabilitation facility. We used a battery of approaches to control for confounding, including regression adjustment and propensity scores.

\section{Methods \\ Data and Cohort Creation}

This study was approved by the Dartmouth Committee for Protection of Human Subjects. The data were anonymized and de-identified prior to use and therefore no informed consent was required. We used 100\% of Medicare Denominator file and corresponding Medicare inpatient and outpatient claims Parts A and B 2007-2012 (MedPAR, Carrier and Outpatient files) to select patients with unruptured cerebral aneurysm diagnosis. Aneurysm patients were identified based on one or more inpatient or outpatient diagnoses (ICD-9 diagnosis code 437.3) between 2007 and 2012. For cohort inclusion, patients were required to 1) be continuously enrolled in fee-for-service Medicare Parts A and $\mathrm{B}$ for 12 months before index diagnosis, and 2) be 65 years or older at the time of index diagnosis.

\section{Intervention}

We used ICD-9-CM codes to identify patients with unruptured cerebral aneurysms (ICD-9-CM code 437.3) who underwent clipping (ICD-9-CM code 39.51) or coiling (ICD-9-CM code 39.52 [along with a code 88.41 and no code 39.51 during the same hospitalization], 39.72, 39.75, 39.76 39.79) between 2007 and 2012. For patients with multiple interventions, only the first one was included in the final cohort. A neurosurgeon was considered to have a dual practice (open and endovascular) when he/she practiced both approaches, with more than $10 \%$ of the total case volume (based on Medicare data) consisting of the secondary approach.

\section{Outcome Variables}

The primary outcome was 1 -year postprocedure mortality. Secondary outcomes were LOS during the initial hospitalization, rate of discharge to a rehabilitation facility, and rate of 30-day postdischarge readmission.

\section{Covariates}

Sex-age categories $(65-69,70-74,75-79,80-84$, and 85-99 years) were created, as well as 5 ethnicity and race categories (Asian, Black, Hispanic, Native American, and other, with white being the excluded variable). The enroll- ee's ZIP code was used to match to 2010 Census data on income and poverty. We included the ZIP-level poverty rate separately from the income variable, to reflect the differing distribution of income within the ZIP code.

Comorbidities, diagnosed (in more than 2 outpatient and/or 1 inpatient encounters) at any time in the 12-month look-back (before the intervention), for which outcomes were adjusted (Table S1) included hypertension, myocardial infarction, cardiac arrhythmia, congestive heart failure, hyperlipidemia, coagulopathy, hypertension, ischemic stroke, peripheral vascular disease, chronic obstructive pulmonary disease (COPD), other pulmonary disease, diabetes, obesity, alcohol abuse, malignancy, and dementia.

\section{Statistical Analysis}

To compare outcomes between hybrid neurosurgeons and physicians performing only endovascular coiling (clipping or coiling) therapies, we used several methods to address measured confounding, one of which was based on propensities. Initially, to compare death at 1 year postoperatively, 30-day readmission, and discharge to rehabilitation between hybrid neurosurgeons and proceduralists performing only endovascular coiling, we employed a multivariable logistic regression, including all the covariates listed above, the proceduralist's case volume, and the proceduralist's specialty (neurosurgery, radiology, and other). To account for clustering of observations within physicians, we employed a sandwich variance based on an exchangeable correlation matrix. For LOS, we employed the corresponding versions of multiple linear regression models. In sensitivity analysis, we repeated this approach after logarithmic transformation of LOS. The results were similar and are therefore not reported further.

To further control for confounding, we used regression models with adjustment (stratification) by quantiles (we chose the number of quantiles to be 20) of propensity score. To derive the propensity of undergoing treatment performed by a hybrid neurosurgeon, we developed a prediction model using logistic regression, based on the covariates described above. To account for clustering of observations within physicians, we employed a sandwich variance based on an exchangeable correlation matrix.

Additionally, in sensitivity analysis, we examined the effect of various other definitions of hybrid neurosurgeons (defined in our primary analysis as neurosurgeons with more than $10 \%$ of their practice consisting of the secondary procedure) on outcomes (based on Medicare data). We repeated all the above analyses twice: once considering as hybrid neurosurgeons to be those with at least one secondary procedure (e.g., if a neurosurgeon was performing mainly coiling, he/she was considered a hybrid neurosurgeon if he/she had performed one clipping procedure), and again considering hybrid neurosurgeons to be those with more than 5 secondary procedures. Additionally, we applied these 3 definitions only for the surgeon's case volume in the year prior to the intervention in question and repeated the prespecified analyses. Our results were remarkably robust to all of these sensitivity analyses. The direction of the observed associations was identical in these different iterations, and therefore these results are not reported further. The relationship of annual case volume and 1-year 
mortality was additionally examined with the use of a locally weighted scatterplot smoothing graph. We set the smoothing parameter (i.e., effective degrees of freedom) at 3.0. Lastly, in prespecified subgroup analyses, we examined the differences in outcomes among various specialties (neurosurgeons, radiologists, other), controlling for all covariates listed previously, including case volume. Age and pretreatment comorbidity burden (as reflected by the Charlson Comorbidity Index) were integrated in the initial regressions, and also used for additional stratified analysis. Our results were robust in all these additional analyses.

Given that we had 11,716 patients from 3246 combined practitioners, and approximately $10 \%$ were hybrid practitioners, we had an $80 \%$ power to detect a difference in mortality as small as $2.5 \%$ among patients treated by hybrid and nonhybrid proceduralists, e.g., $4.5 \%$ vs $7.0 \%$ at an $\alpha$-level of 0.05 . Patients with missing data (3\% of poverty and income) were excluded from further analysis. All probability values were the result of 2-sided tests. SAS (version 9.4, SAS Institute), and the 64-bit version of R.2.12.2 (R Foundation for Statistical Computing) were used for statistical analysis.

\section{Results}

\section{Patient Characteristics}

Between 2007 and 2012, there were 11,716 Medicare patients who underwent endovascular coiling for unruptured cerebral aneurysms and met the inclusion criteria for the study. From these patients, 1186 (10.1\%) underwent treatment performed by a hybrid neurosurgeon, whereas $10,530(89.9 \%)$ underwent treatment by physicians performing exclusively endovascular coiling. The respective distribution of exposure variables between the 2 types of proceduralists can be found in Table 1 .

\section{Mortality}

Among patients undergoing endovascular coiling, 93 $(4.9 \%)$ deaths were recorded in the 1 st year after treatment by a hybrid neurosurgeon, and 684 (6.5\%) after treatment by interventionalists who only performed coiling (Table 2). As demonstrated in Table 3 , being treated by a hybrid neurosurgeon was not associated with increased 1-year mortality (OR 0.78; 95\% CI 0.54-1.12) in the unadjusted analysis. Similarly, adjusting for confounders with a multivariable logistic regression model (Table 3 ) demonstrated a lack of association of combined practice with 1-year mortality (OR 0.77; 95\% CI 0.49-1.22), which persisted after propensity score adjustment (OR 0.84; 95\% CI $0.58-$ 1.23) (Table 3).

Higher procedural volume was associated with lower mortality, regardless of the focus of the proceduralist's practice. Performing 10-20 (OR 0.55; 95\% CI 0.41-0.75),

TABLE 1. Patient characteristics*

\begin{tabular}{|c|c|c|c|}
\hline Variable & Treatment by Nonhybrid Proceduralists & Treatment by Hybrid Neurosurgeons & z Value \\
\hline Mean age in yrs & $72.4 \pm 5.2$ & $72.2 \pm 5.1$ & -1.5 \\
\hline Male sex & $2,506(23.9)$ & $386(20.5)$ & -3.2 \\
\hline African American race & $734(7.0)$ & $132(7.0)$ & 0.1 \\
\hline Mean income $†$ & $\$ 46,700 \pm 16,900$ & $\$ 48,268 \pm 17,626$ & 3.5 \\
\hline Poverty† & $952(9.4)$ & $162(9.0)$ & -2.4 \\
\hline \multicolumn{4}{|l|}{ Comorbiditiesł } \\
\hline Hypertension & $5,529(52.5)$ & $1,008(53.4)$ & 0.8 \\
\hline Hyperlipidemia & $2,383(22.6)$ & $473(25.1)$ & 2.3 \\
\hline COPD & $267(2.5 \%)$ & $49(2.6)$ & 0.2 \\
\hline Myocardial infarction & $1,533(14.6)$ & $220(11.7)$ & -3.3 \\
\hline Cardiac arrhythmia & $804(7.6)$ & $123(6.5)$ & -1.7 \\
\hline Coagulopathy & $102(1.0)$ & $12(0.6)$ & -1.4 \\
\hline Renal insufficiency & $381(3.6)$ & $57(3.0)$ & -1.3 \\
\hline Congestive heart failure & $461(4.4)$ & $64(3.4)$ & -2.0 \\
\hline Pulmonary disease§ & $261(2.5)$ & $53(2.8)$ & 0.8 \\
\hline Obesity & $83(0.8)$ & $18(1.0)$ & 0.7 \\
\hline Alcohol abuse & $33(0.3)$ & - & \\
\hline Dementia & $138(1.3)$ & $13(0.7)$ & -2.3 \\
\hline Ischemic stroke & $1,260(12.0)$ & $224(11.9)$ & -0.1 \\
\hline Diabetes & $1,529(14.5)$ & $273(14.5)$ & -0.1 \\
\hline Peripheral vascular disease & $1,357(12.9)$ & $277(14.7)$ & 2.1 \\
\hline Malignancy & $805(7.6)$ & $121(6.4)$ & -1.9 \\
\hline
\end{tabular}


TABLE 2. Outcomes*

\begin{tabular}{lccc}
\hline \multicolumn{1}{c}{ Variable } & Treatment by Nonhybrid Proceduralists & Treatment by Hybrid Neurosurgeons & $p$ Value \\
\hline 1-yr mortality & $684(6.5)$ & $93(4.9)$ & 0.683 \\
\hline 30-day readmission & $1,489(14.1)$ & $271(14.4)$ & 0.282 \\
\hline Discharge to rehabilitation & $525(5.0)$ & $90(4.8)$ & 0.351 \\
\hline Mean LOS in days & $3.5 \pm 5.1$ & $3.1 \pm 4.5$ & 0.172 \\
\hline
\end{tabular}

* Values are crude numbers and percentages in parentheses, unless otherwise indicated. Mean values are presented as the mean \pm SD.

20-50 (OR 0.52; 95\% CI 0.38-0.71), or more than 50 procedures per year (OR 0.42 ; 95\% CI $0.27-0.65)$ was associated with decreased mortality in comparison with lowvolume proceduralists (fewer than 10 coiling procedures per year). Figure 1 demonstrates a graphic representation of the association of proceduralist's case volume with 1 -year mortality of patients undergoing endovascular coiling for unruptured cerebral aneurysms.

When controlling for case volume the specialty of the proceduralist had no association with mortality. There was no difference between neurosurgeons and radiologists (OR 0.85; 95\% CI 0.59-1.22), or between neurosurgeons and other proceduralists (OR 0.86 ; $95 \%$ CI $0.53-1.39$ ) performing the operation.

\section{Length of Stay}

Among patients undergoing endovascular coiling, the average LOS was $3.1 \pm 4.5$ days $( \pm \mathrm{SD})$ after treatment performed by a combined proceduralist, and $3.5 \pm 5.1$ days after treatment by interventionalists who only performed coiling (Table 2). As demonstrated in Table 3, being treated by a combined proceduralist was not associated with increased LOS (adjusted difference $0.31 ; 95 \%$ CI -0.38 to 0.99 ) in the unadjusted analysis. Similarly, adjusting for confounders with a multivariable logistic regression model (Table 3) demonstrated a lack of association of combined practice with LOS (adjusted difference 0.42; 95\% CI -0.24 to 1.08 ), which persisted after propensity score adjustment (adjusted difference $0.41 ; 95 \%$ CI -0.26 to 1.09) (Table 3).

\section{Discharge to a Rehabilitation Facility}

Among patients undergoing endovascular coiling, 90 (4.8\%) patients were discharged to rehabilitation (Table 2) after treatment by a combined proceduralist, and 525 (4.9\%) after treatment by interventionalists who only performed coiling. As demonstrated in Table 3, being treated by a combined proceduralist was not associated with increased rate of discharge to rehabilitation (OR 0.97; 95\% CI 0.64-1.46) in the unadjusted analysis. Similarly, adjusting for confounders with a multivariable logistic regression model (Table 3) demonstrated a lack of association of combined practice with rate of discharge to rehabilitation (OR 0.95; 95\% CI 0.57-1.58), which persisted after propensity score adjustment (OR 1.00; 95\% CI 0.66-1.51) (Table 3).

\section{0-Day Readmission}

Among patients undergoing endovascular coiling, 271
(14.4\%) were readmitted within 30 days of discharge (Table 2) after treatment by a combined proceduralist, and $1489(14.1 \%)$ after treatment by interventionalists who only performed coiling. As demonstrated in Table 3, being treated by a combined proceduralist was not associated with a higher rate of 30-day readmission (OR 1.04; 95\% CI 0.81-1.35) in the unadjusted analysis. Similarly, adjusting for confounders with a multivariable logistic regression model demonstrated a lack of association of combined practice with 30 -day readmission (OR $1.03 ; 95 \%$ CI $0.75-1.42$ ), which persisted after propensity score adjustment (OR 1.07; 95\% CI 0.83-1.38) (Table 3).

\section{Discussion}

In a cohort of elderly patients undergoing endovascular surgical treatment of unruptured cerebral aneurysms, we did not identify an association of treatment by hybrid neurosurgeons with 1-year mortality, LOS, discharge to rehabilitation, or 30-day readmission. Proceduralists with higher case volume had improved outcomes, regardless of the focus of their practice or specialty. These results were consistent when using propensity score adjustment and controlling for clustering at the physician level. Regional coiling rates are highly variable, ranging from $35 \%$ in Modesto, CA, to $98.6 \%$ in Tacoma, WA, ${ }^{1}$ secondary to the varying expertise and training of the respective providers. Radiologists, neurosurgeons, and other proceduralists perform endovascular coiling. From these, dually trained neurosurgeons, using both open and endovascular techniques, is the fastest growing group. ${ }^{1}$

However, some argue that these skill sets are not compatible, and therefore proceduralists performing only endovascular coiling have superior outcomes. They claim that hybrid neurosurgeons have a diffuse practice and never become experts in this procedure. On the contrary, others believe that dually trained neurosurgeons provide an unbiased and well-considered assessment of which therapy would be indicated in a given patient. ${ }^{\mathbf{1 , 6}}$ De Vries and Boogaarts, both hybrid neurosurgeons, demonstrated low periprocedural complications in their personal series. ${ }^{6}$ They treated $80 \%$ of their patients with endovascular means. These interventions were associated with combined in-hospital morbidity and mortality of $1.4 \%$. However, the lack of a comparison group of only endovascular proceduralists is making these results difficult to generalize.

In our study of unruptured aneurysms, we intentionally addressed these limitations. First, we created a cohort of almost all elderly patients in the United States, giving 

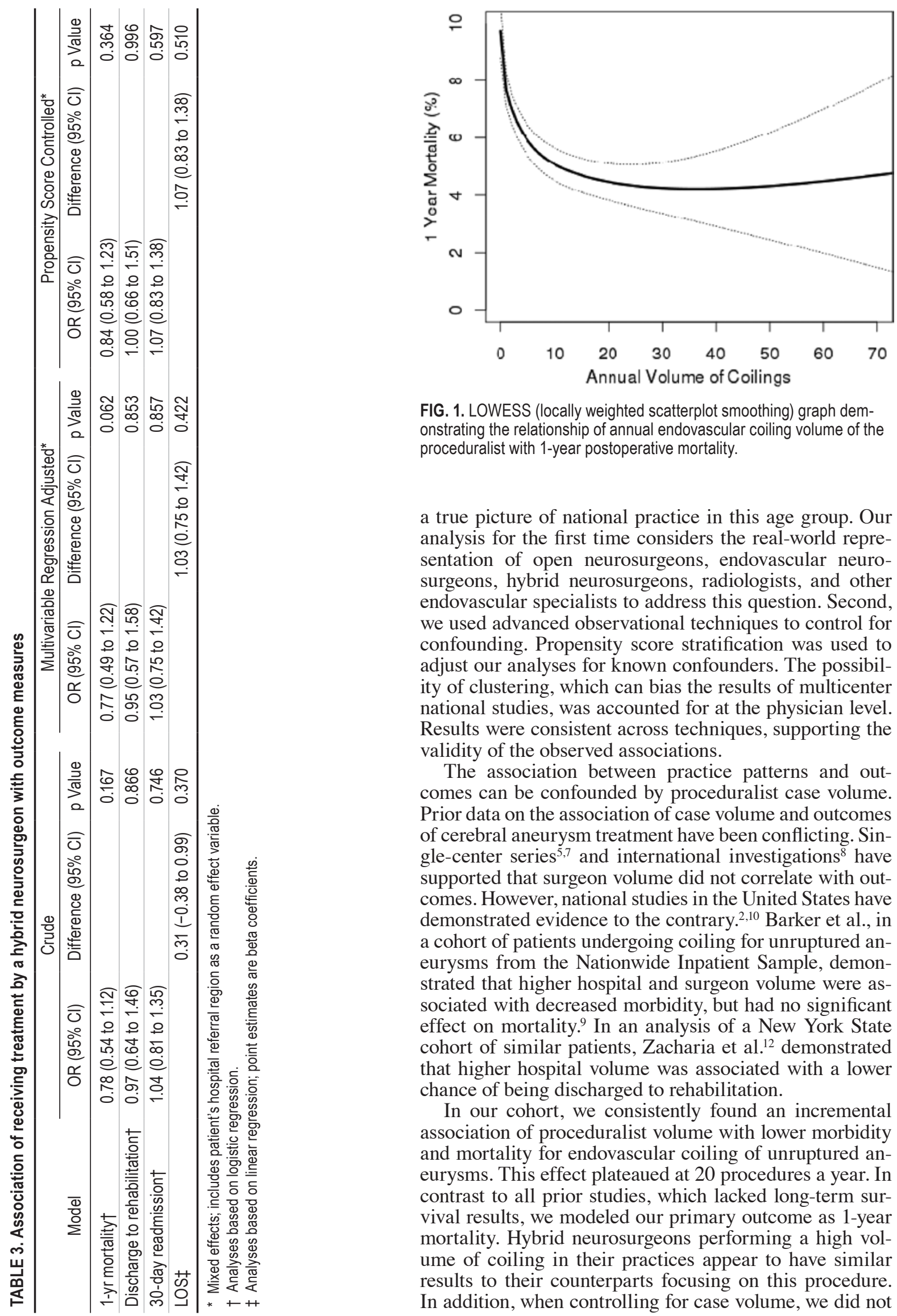

FIG. 1. LOWESS (locally weighted scatterplot smoothing) graph demonstrating the relationship of annual endovascular coiling volume of the proceduralist with 1-year postoperative mortality.

a true picture of national practice in this age group. Our analysis for the first time considers the real-world representation of open neurosurgeons, endovascular neurosurgeons, hybrid neurosurgeons, radiologists, and other endovascular specialists to address this question. Second, we used advanced observational techniques to control for confounding. Propensity score stratification was used to adjust our analyses for known confounders. The possibility of clustering, which can bias the results of multicenter national studies, was accounted for at the physician level. Results were consistent across techniques, supporting the validity of the observed associations.

The association between practice patterns and outcomes can be confounded by proceduralist case volume. Prior data on the association of case volume and outcomes of cerebral aneurysm treatment have been conflicting. Single-center series $s^{5,7}$ and international investigations ${ }^{8}$ have supported that surgeon volume did not correlate with outcomes. However, national studies in the United States have demonstrated evidence to the contrary. ${ }^{2,10}$ Barker et al., in a cohort of patients undergoing coiling for unruptured aneurysms from the Nationwide Inpatient Sample, demonstrated that higher hospital and surgeon volume were associated with decreased morbidity, but had no significant effect on mortality. ${ }^{9}$ In an analysis of a New York State cohort of similar patients, Zacharia et al. ${ }^{12}$ demonstrated that higher hospital volume was associated with a lower chance of being discharged to rehabilitation.

In our cohort, we consistently found an incremental association of proceduralist volume with lower morbidity and mortality for endovascular coiling of unruptured aneurysms. This effect plateaued at 20 procedures a year. In contrast to all prior studies, which lacked long-term survival results, we modeled our primary outcome as 1-year mortality. Hybrid neurosurgeons performing a high volume of coiling in their practices appear to have similar results to their counterparts focusing on this procedure. In addition, when controlling for case volume, we did not 
observe any differences in outcomes between neurosurgeons, radiologists, or other proceduralists performing endovascular coiling. These results highlight that the debate should not focus on the specialty of the physicians performing these procedures but on their case volume, given the increasing trend of endovascular coiling being performed by low-volume, inexperienced proceduralists. ${ }^{3}$

This study has several limitations common to administrative databases. First, this is an observational investigation, and there is still a possibility of residual confounding. We used multiple techniques (propensity score stratification, hospital referral region random effects), yielding consistent results to account for confounders. Second, coding inaccuracies can affect our estimates. However, coding for procedures is rarely inaccurate, given that it is a revenue generator and is under scrutiny by payers.

Third, claims data do not provide metrics on the postoperative neurological status of the patients (i.e., modified Rankin Scale score), chronic pain, or quality of life. Therefore, we cannot analyze the difference in surgeon expertise in regard to these measures. Alternatively, this question can be answered by the creation of large, long-term registries, with such efforts currently underway (http://www.neuro point.org/NPA\%20N2QOD.html). Quality of life outcome measures or patient satisfaction metrics could be used instead in future prospective investigations. Fourth, findings among this older, American population may not be generalizable to younger or otherwise dissimilar populations. Our definition of combined practice is only based on Medicare data, and therefore does not reflect practice patterns among younger patients. However, it is less likely that a surgeon would perform only one approach (i.e., endovascular) for all Medicare patients in the span of 6 years of the study and follow a different practice pattern in younger patients. Fifth, we cannot analyze any potential selection biases on aneurysms treated by open or endovascular means. However, the present analysis focuses only on endovascular approaches. Additionally, we expect that surgeons would treat similar aneurysms with clipping (mostly anterior circulation) or coiling (mostly posterior circulation). Regardless of whether they have a combined practice. Sixth, we could not analyze, based on the available data, the percentage of nonaneurysmal cases for proceduralists performing only endovascular approaches. Lastly, causal inference is hard to establish based on observational data, even when using advanced observational techniques.

\section{Conclusions}

The impact of combined practices on the outcomes of unruptured cerebral aneurysm coiling remains an issue of debate. In a cohort of elderly patients undergoing endovascular surgical treatment of unruptured cerebral aneurysms, we did not identify an association of treatment by hybrid neurosurgeons with 1-year mortality, LOS, discharge to rehabilitation, or 30-day readmission. Proceduralists with higher case volumes had improved outcomes, regardless of the focus of their practice or their specialty. Future comparative effectiveness studies will likely need to be based on prospective registries, using quality outcome metrics, when determining which approach is best.

\section{Acknowledgments}

We thank Jon Skinner, $\mathrm{PhD}$, for his thoughtful review and recommendations on the manuscript. This work was supported by grants from the National Institute on Aging (P01-AG19783), the National Institutes of Health Common Fund (U01-AG046830), and the National Center for Advancing Translational Sciences (NCATS) of the NIH (Dartmouth Clinical and Translational Science Institute-UL1TR001086). The funders had no role in the design or execution of the study.

\section{References}

1. Bekelis K, Goodney RP, Dzebisashvili N, Goodman DC, Bronner KK: Variation in the care of surgical conditions: cerebral aneurysms, in Goodney PR, Dzebisashvili N, Goodman DC, et al. (eds): Variations in the Care of Surgical Conditions. Hanover, NH: Dartmouth College, 2015

2. Brinjikji W, Kallmes DF, Lanzino G, Cloft HJ:

Hospitalization costs for endovascular and surgical treatment of ruptured aneurysms in the United States are substantially higher than Medicare payments. AJNR Am J Neuroradiol 33:1037-1040, 2012

3. Brinjikji W, Lanzino G, Kallmes DF, Cloft HJ: Cerebral aneurysm treatment is beginning to shift to low volume centers. J Neurointerv Surg 6:349-352, 2014

4. Brisman JL, Song JK, Newell DW: Cerebral aneurysms. N Engl J Med 355:928-939, 2006

5. Chang TR, Kowalski RG, Carhuapoma JR, Tamargo RJ, Naval NS: Impact of case volume on aneurysmal subarachnoid hemorrhage outcomes. J Crit Care 30:469472, 2015

6. de Vries J, Boogaarts HD: Treatment of patients with ruptured aneurysm by neurosurgeons that perform both open surgical and endovascular techniques is safe and effective: results of a single centre in Europe. Acta Neurochir (Wien) 156:1259-1266, 2014

7. Goldschlager T, Selvanathan S, Walker DG: Can a "novice" do aneurysm surgery? Surgical outcomes in a low-volume, non-subspecialised neurosurgical unit. J Clin Neurosci 14:1055-1061, 2007

8. Hattori N, Katayama Y, Abe T: Case volume does not correlate with outcome after cerebral aneurysm clipping: a nationwide study in Japan. Neurol Med Chir (Tokyo) 47:95-101, 2007

9. Hoh BL, Rabinov JD, Pryor JC, Carter BS, Barker FG II: Inhospital morbidity and mortality after endovascular treatment of unruptured intracranial aneurysms in the United States, 1996-2000: effect of hospital and physician volume. AJNR Am J Neuroradiol 24:1409-1420, 2003

10. Johnston SC: Effect of endovascular services and hospital volume on cerebral aneurysm treatment outcomes. Stroke 31:111-117, 2000

11. Molyneux A, Kerr R, Stratton I, Sandercock P, Clarke M, Shrimpton J, et al: International Subarachnoid Aneurysm Trial (ISAT) of neurosurgical clipping versus endovascular coiling in 2143 patients with ruptured intracranial aneurysms: a randomised trial. Lancet 360:1267-1274, 2002

12. Zacharia BE, Bruce SS, Carpenter AM, Hickman ZL, Vaughan KA, Richards C, et al: Variability in outcome after elective cerebral aneurysm repair in high-volume academic medical centers. Stroke 45:1447-1452, 2014

\section{Disclosures}

The authors report no conflict of interest concerning the materials or methods used in this study or the findings specified in this paper. 


\section{Author Contributions}

Conception and design: Bekelis. Acquisition of data: Bekelis, Gottlieb, Su, MacKenzie. Analysis and interpretation of data: all authors. Drafting the article: Bekelis. Critically revising the article: Gottlieb, Labropoulos, Su, Tjoumakaris, Jabbour, MacKenzie. Reviewed submitted version of manuscript: Bekelis, Labropoulos, Tjoumakaris, Jabbour, MacKenzie. Approved the final version of the manuscript on behalf of all authors: Bekelis Statistical analysis: Bekelis, Gottlieb, Su, MacKenzie. Administrative/technical/material support: Labropoulos. Study supervision: Bekelis, MacKenzie.

\section{Supplemental Information}

Online-Only Content

Supplemental material is available with the online version of the article.

Table S1. http://thejns.org/doi/suppl/10.3171/2015.11. JNS151725.

\section{Correspondence}

Kimon Bekelis, Section of Neurosurgery, Dartmouth-Hitchcock Medical Center, One Medical Center Dr., Lebanon, NH 03755. email: kbekelis@gmail.com. 